\title{
COMPTEL OBSERVATIONS OF X-RAY BINARIES
}

\author{
A. F. IYUDIN \\ MPI für extraterrestrische Physik, Garching, FRG
}

The COMPTEL experiment on the Compton Gamma-Ray Observatory measures $\gamma$-radiation in the energy range from 0.75 to $30 \mathrm{MeV}$ [10]. X-ray binaries form one category of potential $\gamma$-ray sources. Up to now only two $\mathrm{X}$-ray binaries, Cyg X-1 and Nova Per, have been seen by COMPTEL.

Cyg X-1. Preliminary results were reported by [6] and indicate that (i) there was no evidence for any hardening of the spectrum near $1 \mathrm{MeV}$, (ii) the plasma temperature suggested by a Wien spectral model was much higher than that implied by hard X-ray observations. This latter conclusion seems to require a revision in the standard spectral model for Cyg X-1.

The $\gamma$-ray flux from Cyg X-1 is constant up to several MeV. The time history of the flux in the $0.75 \ldots 2.0 \mathrm{MeV}$ energy range shows that there are no significant variations near $1 \mathrm{MeV}$. The average photon spectrum derived from all observations up to 1994 July shows evidence for significant emission extending well above $2 \mathrm{MeV}$, with a data point in the $2 \ldots 5 \mathrm{MeV}$ range at $4.3 \sigma$ significance. However, the observed flux near $1 \mathrm{MeV}$ is significantly below that reported by HEAO-3 and several other balloon observations [6]. The COMPTEL spectral data show a broad line-like feature around $4 \mathrm{MeV}$ with the best fit of a single power law plus Gaussian. If we assume that the Gaussian represents a single broadened nuclear line of ${ }^{12} \mathrm{C}^{*}$, then the width of $1.38 \mathrm{MeV}$ demands a temperature of $\sim 210^{12} \mathrm{~K}$ [7].

Nova Per 1992. The X-ray transient GRO J0422+32 is also known as Nova Per 1992 [8]. COMPTEL observed this object twice during ToO viewing periods 1992 August $12 \ldots 20$ and 1992 September $1 . .17$, and then later in 1993...94. First results on the COMPTEL detection of $\gamma$-ray emission from this source were reported by [4], [5]. During the first observation, which started when the X-ray flux was at maximum, Nova Per was detected with flux in the $1 \ldots 2 \mathrm{MeV}$ energy range that was higher than expected, based on extrapolation of the standard Comptonisation model [11] fits to the contemporaneous OSSE [2] and SIGMA [9] data. Nova Per was seen up to $\sim 2 \mathrm{MeV}$ during a primary outburst in 1992 Aug... Sep by COMPTEL. There are indications that this binary had secondary outbursts in visual

277

A. Evans and J. H. Wood (eds.), Cataclysmic Variables and Related Objects, 277-278.

(C) 1996 Kluwer Academic Publishers. Printed in the Netherlands. 
light, with period $\sim 125 \mathrm{~d}$. The spectrum also shows large deviations from the standard Comptonisation model in the $\mathrm{MeV}$ energy range.

Preliminary results of the continued analysis of all available COMPTEL data for the period $1992 \ldots 94$ can be summarised as follows: $\gamma$-ray emission at $1 \ldots 2 \mathrm{MeV}(\sim 3 \sigma)$ was detected during the main outburst [4], [5] and later during viewing period (VP) 325 (1994 April $22 \ldots$ May 5) with $\sim 4.3 \sigma$ significance in the $1.5 \ldots 2.1 \mathrm{MeV}$ band. VP325 presumably corresponds to the time of the nova recurrent optical activity (time of VP325 - time of main outburst $=5$ periods of $\sim 125 \mathrm{~d}$, as derived by [1] and [3]). For all VPs for which no activity of Nova Per 1992 was observed only upper limits on the $\gamma$-ray flux, which are significantly $(\gtrsim 3 \sigma)$ lower than the flux in the same energy intervals during active periods, were derived.

A spectral fit of the available data was performed with the standard Comptonisation model of [11], and with the more generalised Comptonisation model of [12], and resulted in electron temperatures $\sim 100 \mathrm{keV}$ [5]. These results are in accordance with the higher temperatures $(\sim 200 \mathrm{keV})$ found by COMPTEL for Cyg X-1 [6]. The quality of the fits with the use of the Wien type spectrum were generally not very good and show significant deviations from the Comptonisation models at the higher energy intervals $(300 \ldots 700 \mathrm{keV})$ of SIGMA (5.8 $\sigma$ deviation) [9] and at the COMPTEL energies $(2.8 \sigma)[5]$.

Conclusion: Both objects (i) belong to the systems with a black hole companion and (ii) show a large deviation in the spectrum from the Compton model fit. Nova Per shows excess emission above the continuum in relatively narrow energy bands. The presence of nonthermal emission processes in both objects seems unavoidable.

Acknowledgement. This work was presented on behalf of the COMPTEL collaboration.

\section{References}

1. Callanan, P.J., Garcia, M.R., McClintock, J.E., et al., 1995, Ap. J., 441, 786

2. Cameron, R.A., et al., 1992, IAUC 5587

3. Chevalier, C., Ilovaisky, S.A., 1994, preprint No. 83, Observatoire de Haute-Provence

4. van Dijk, R., et al., 1994, in AIP 304 Conf. Proceed., "The Second Compton Symposium”, eds. C. E. Fichtel, N. Gehrels, J. P. Norris, (New York: AIP), p197

5. van Dijk, R., Bennett, K., Collmar, W., et al,, 1995, A\&A, 296, L33

6. McConnell, M.L., Forrest, D., Ryan, J., et al., 1994, Ap. J., 424, 933

7. McConnell, M.L., et al., 1995, presented at the Third Compton Symposium, München

8. Paciesas, W.C., et al., 1993, in AIP 304 Conf. Proceed., "The Second Compton Symposium", eds. C. E. Fichtel, N. Gehrels, J. P. Norris, (New York: AIP), p365

9. Roques, J.P., Bouchet, L., Jourdain, E., et al., 1994, Ap. J. Supp., 92, 451

10. Schönfelder, V., Aarts, H., Bennett, K., et al., 1993, Ap. J. Supp., 86, 657

11. Sunyaev, R.A., Titarchuk, L.G., 1980, A\&A, 86, 121

12. Titarchuk, L., 1994, Ap. J., 434, 570 